\title{
Pericarditis in Children and Young Adults: A Monocentric Study
}

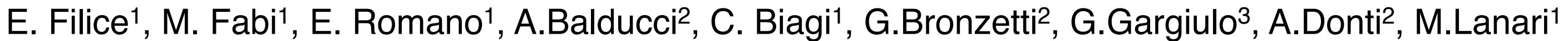 \\ 1: Pediatria d'Urgenza, Pronto Soccorso e Osservazione Breve Intensiva, Policlinico Sant'Orsola-Malpighi, Bologna 2: Cardiologia Pediatrica e dell'età Evolutiva, Policlinico Sant'Orsola-Malpighi, Bologna \\ 3: Cardiochirurgia Pediatrica e dell'età Evolutiva, Policlinico Sant'Orsola-Malpighi, Bologna}

\section{BACKGROUND}

Pericarditis is an acute inflammation of the pericardium

with or without pericardial effusion. Pericarditis in children accounts for $5 \%$ of pediatric presentations to emergency departments for chest pain 1 . Male gender and age between 16 to 65 increase the risk with a RR $2.02^{2}$.

USA data documented that idiopathic and viral etiologies are more common in males $(71 \%)$ at a mean age 14.5 years ${ }^{2}$.

Diagnosis in children requires the same criteria as in adults: chest pain, pericardial rubs, ECG changes with new widespread ST elevation or PR depression, pericardial effusion. Additional signs and symptoms may be present according to the underlying disease ${ }^{3}$. Scarce data are available in literature about the etiology in pediatric age.

\section{AIM}

To evaluate etiology, features

at the onset, and treatment of

pericarditis in our population.
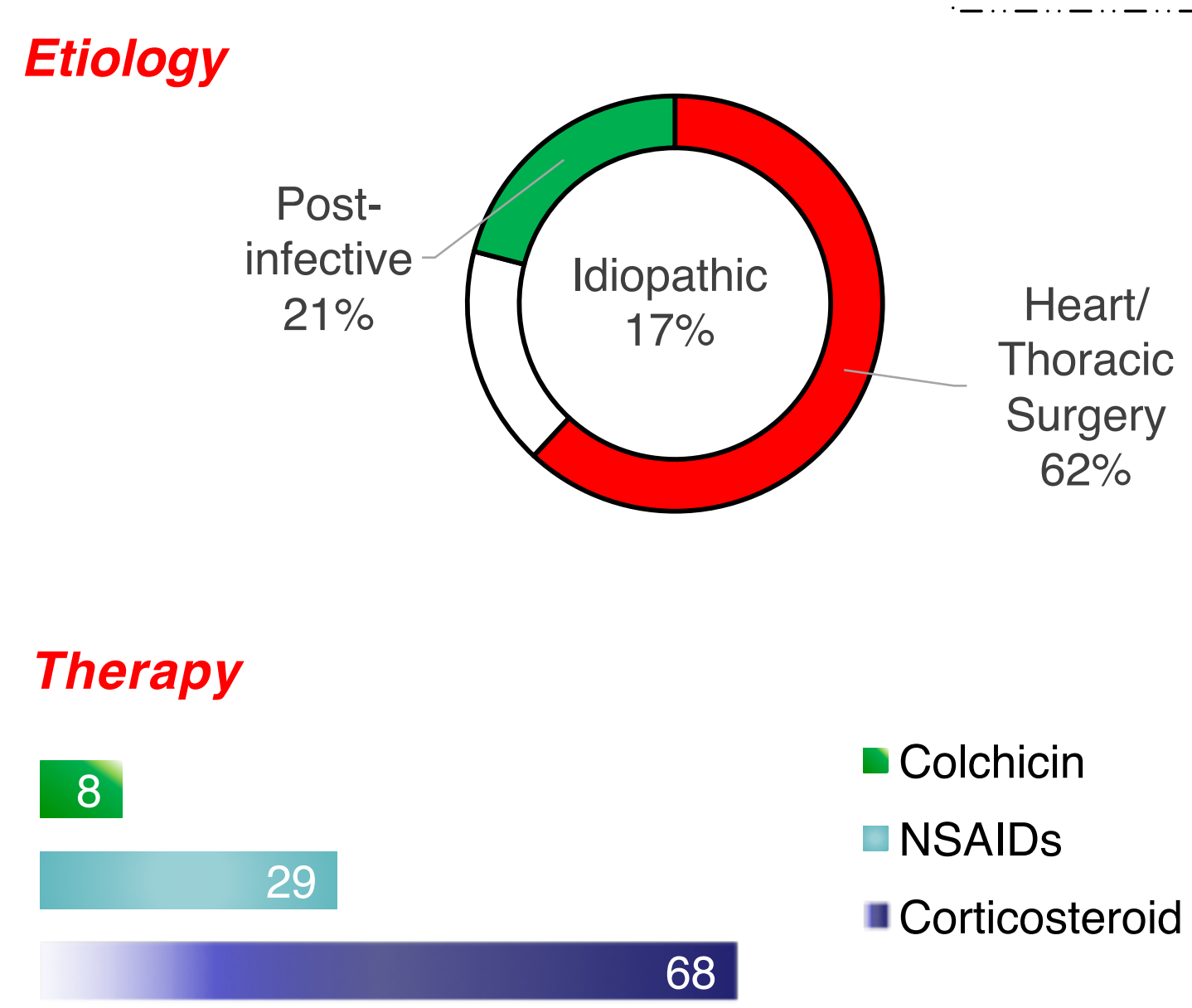

\section{Clinical signs and radiology findings}

80

70

60

50

40 30

20 10 0

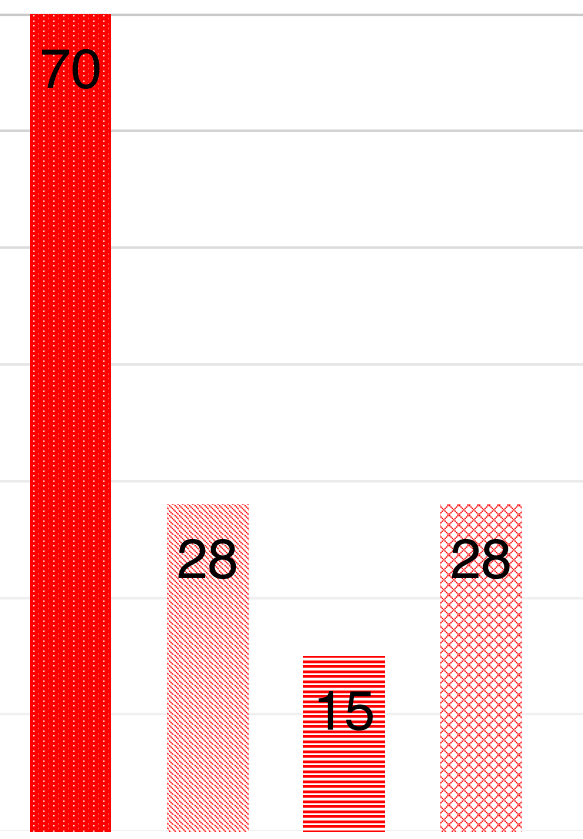

\section{METHOD}

Monocentric retrospective study of patients

diagnosed with pericarditis between January 2013 and December 2017. Diagnosis was made according to 2015 ESC Guidelines ${ }^{3}$.

\section{RESULTS}

We included 120 patients

[mean age 10 years, 74

males]. 65 (54\%) underwent heart or thoracic surgery in

the previous month, 22

$(18 \%)$ had suffered from an

upper respiratory tract infection 2 to 3 weeks earlier, $18(15 \%)$ were diagnosed with idiopathic pericarditis [more often $(p<0,01)$ among patients aged 18 to 23]. 37 (31\%) reported chest pain, $25(21 \%)$ fever.

- Chest pain

- Fever

- Tamponade signs

$\square$ Pericardial rubs

- Pericardial effusion $<10 \mathrm{~mm}$

- Pericardial effusion $10-20 \mathrm{~mm}$

豆Pericardial effusion $>20 \mathrm{~mm}$

Pleural effusion

\section{CONCLUSION}

In our cohort, idiopathic and post-infective etiologies accounted for $30 \%$, the prevalence of the idiopathic being significantly higher in the age group of 18 to 23 .

Chest pain and fever were the most frequent symptoms at the onset.

\section{SOURCES}

1: Imazio M. Contemporary management of pericardial diseases. Curr Opin Cardiol 2012; 27:308-317.

2: Shakti D, Hehn R, Gauvreau K, Sundel RP, Newburger JW. Idiopathic pericarditis and pericardial effusion in children: contemporary epidemiology and management. $J$ Am Heart Assoc. 2014 Nov 7;3(6):e001483

3: 2015 ESC Guidelines for the diagnosis and management of pericardial diseases. The Task Force for the Diagnosis and Management of Pericardial Diseases of the European Society of Cardiology (ESC); European Heart Journal (2015) 36, 2921-2964.

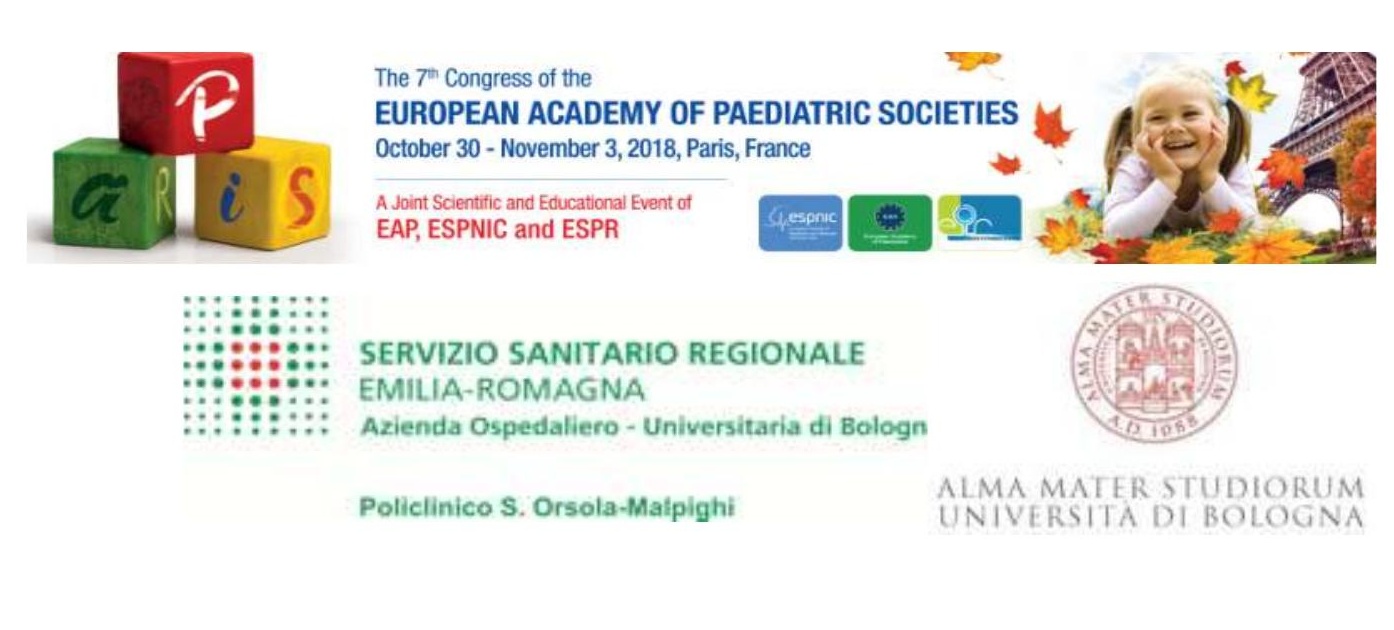

